Journal of Affective DisordersVolume 246, 1 March 2019, Pages 327-328

\title{
Comments on "Affective instability in those with and without mental disorders: A case control study" by Marwaha et al.(Letter)
}

- Cornejo-Rojas, D.A. ${ }^{\text {EEmail Author, }}$

- Castillo-Soto, A.a, ,

- Araujo-Castillo, R.V.

- View Correspondence (jump link)

- aSchool of Medicine, Universidad Peruana de Ciencias Aplicadas, Alameda San Marcos cuadra 2, Chorrillos, Lima, Peru

- 'Sociedad Cientifica de Estudiantes de Medicina UPC, Alameda San Marcos cuadra 2, Chorrillos, Lima, Peru

\begin{abstract}
View references (4)
This letter has the purpose to comment the article by Marwaha et al. regarding affective instability and mental disorders. We wish to highlight the importance to report the proper measures of association in case-control studies, and the impact of adjusting the results when finding associations with possible confounders in the bivariate analysis. (C) 2018 Elsevier B.V.
\end{abstract}

SciVal Topic Prominence

Topic: Affect | Depression | ecological momentary Prominence percentile: 96.738

Author keywords

Affective instabilityCase-control studiesMental disordersMultivariate adjustment

Indexed keywords

EMTREE medical terms:

bivariate analysiscase control studyfalse negative resulthumaninternal consist analysismental diseasemental healthmental instabilitymultivariate analysisnul size

- ISSN: 01650327

- CODEN: JADID 
- Source Type: Journal

- Original language: English

- DOI: 10.1016/j.jad.2018.12.061

- Document Type: Letter

- Publisher: Elsevier B.V. 